\title{
Atmosfera modificada e refrigeração para conservação pós-colheita de camu-camu
}

\author{
Modified atmosphere and refrigeration for postharvest camu-camu
}

\author{
Jacqueline de Oliveira $^{\mathrm{I}^{*}}$ Igor Galvão Silva ${ }^{\mathrm{II}}$ \\ Paula Porrelli Moreira da Silva ${ }^{\mathrm{III}}$ Marta Helena Fillet Spoto ${ }^{\mathrm{I}}$
}

\section{RESUMO}

\begin{abstract}
O presente trabalho tem por objetivo avaliar a conservação pós-colheita de camu-camu, utilizando a refrigeração e a atmosfera modificada propiciada pelos filmes de policloreto de vinila $(P V C)$ e polipropileno biaxialmente orientado de $50 \mu \mathrm{m}$ espessura $\left(B O P P_{50}\right)$ e assim avaliar a durabilidade em relação ao controle (sem embalagem). Os frutos foram mantidos a $5 \pm 1{ }^{\circ} \mathrm{Ce}$ $90 \pm 2 \%$ de umidade relativa, durante 25 dias e avaliados a cada quatro dias de armazenamento quanto à firmeza, $\mathrm{pH}$, sólidos solúveis, acidez titulável, ácido ascórbico, antocianinas, perda de massa e concentração de $\mathrm{CO}_{2}$ da atmosfera no interior da embalagem. O filme de BOPP $P_{50}$ mostrou não ser a melhor alternativa para a conservação de camu-camu, pois a sua baixa permeabilidade aos gases ocasionou o acúmulo de $\mathrm{CO}_{2}$ no interior da embalagem, propiciando o processo fermentativo dos frutos após 13 dias de armazenamento. Os atributos $\mathrm{pH}$, sólidos solúveis, acidez titulável, ácido ascórbico e antocianinas não foram afetados pelos tipos de filmes utilizados, mas sim pelos dias de armazenamento. Os resultados mostraram que a utilização do filme de PVC, juntamente com refrigeração, para conservação pós-colheita de camu-camu, foi eficaz na manutenção da firmeza do fruto e na redução de perda de massa, mantendo os frutos comercialmente viáveis por 21 dias, enquanto o controle e os revestidos por $B O P P_{50}$ se apresentaram inviáveis após 13 dias de armazenamento.
\end{abstract}

Palavras-chave: ácido ascórbico, antocianinas, atmosfera modificada, Myrciaria dubia, pós-colheita.

\section{ABSTRACT}

This study aimed to evaluate the postharvest camucamu by using cooling and modified atmosphere propitiated by the films of polyvinyl chloride (PVC) biaxially oriented polypropylene of $50 \mu \mathrm{m}$ thickness $\left(B O P P_{50}\right)$ to evaluate the durability the control (without packaging). The fruits were kept at $5 \pm 1{ }^{\circ} \mathrm{C}$ and $90 \pm 2 \%$ relative humidity for 25 days and evaluated every four days of storage regarding firmness, $\mathrm{pH}$, soluble solids, titratable acidity, vitamin $C$, anthocyanins, weight loss and $\mathrm{CO}_{2}$ concentration of the atmosphere inside the package. The BOPP ${ }_{50}$ film showed not be the best alternative for the conservation of camu-camu, because its low permeability to gases caused the accumulation of $\mathrm{CO}_{2}$ inside the package, allowing the fermentation of fruits after 13 days of refrigerated storage. Characteristics of $\mathrm{pH}$, soluble solids, titrable acidity, vitamin $C$ and anthocyanins were not affected by the types of films used, but through the days of storage. The results showed that the use of PVC film with cooling to postharvest camu-camu, was effective to maintain the firmness and reduction in weight loss by keeping the fruit commercially viable for 21 days, while control coated BOPP ${ }_{50}$ was unviable with 13 days of storage.

Key words: anthocyanins, ascorbic acid, Myrciaria dubia, modified atmosphere, postharvest.

\section{INTRODUÇÃO}

O camu-camu (Myrciaria dubia), espécie arbórea da família Myrtaceae e nativa da região amazônica, vem sendo cultivado com sucesso no Estado de São Paulo (YUYAMA, 2011). É uma fruta com grande potencial econômico e nutricional, sendo rica em ácido ascórbico, antocianinas, compostos fenólicos e apresenta concentrações consideráveis de carotenoides (ARÉVALO PINEDO, 2007; MYODA et al., 2010).

'Departamento de Agroindústria, Alimentos e Nutrição, Escola Superior de Agricultura "Luiz de Queiroz" (ESALQ), Universidade de São Paulo (USP), Av. Pádua Dias, 11, CP 9, 13418-900, Piracicaba, SP, Brasil. E-mail: jacquelineot@hotmail.com. *Autor para correspondência. ${ }^{\text {II } C e n t r o ~ d e ~ E n e r g i a ~ N u c l e a r ~ n a ~ A g r i c u l t u r a ~(C E N A), ~ U S P, ~ P i r a c i c a b a, ~ S P, ~ B r a s i l . ~}$

IIIDepartamento de Ciências Biológicas, ESALQ, USP, Piracicaba, SP, Brasil. 
O consumo de frutas tropicais não tradicionais é crescente no mercado nacional e internacional, devido ao reconhecimento de seus valores terapêuticos e nutricionais, já que muitas são ricas em compostos bioativos (MYODA et al., 2010; RUFINO et al., 2010). Um alimento com alta capacidade antioxidante como o camu-camu despertou o interesse de consumidores, agricultores e indústrias, além de atrair importadores do Japão, Europa e EUA (ARÉVALO PINEDO, 2007).

Apesar da importância, do aumento da produção e do consumo dessa espécie frutífera, há grande carência de informações sobre o comportamento pós-colheita destes frutos em relação ao potencial e tecnologias de conservação. Tal conhecimento é essencial para ampliar o tempo de armazenamento e comercialização, sem, contudo, alterar características físicas, sensoriais e nutricionais dos frutos. Assim como o camu-camu, a maioria das frutas tropicais apresenta curto período de comercialização após a colheita, devido à intensa atividade metabólica e ao alto conteúdo de água em sua composição química. Segundo CHITARRA \& CHITARRA (2005), dentre as técnicas utilizadas para prolongar a vida-útil de frutos in natura, pode-se citar a utilização de atmosfera modificada e refrigeração, tecnologias que visam minimizar a intensidade do processo vital das frutas por meio da utilização de condições adequadas, que permitam reduzir o metabolismo normal, evitando, assim, a rápida deterioração.

A modificação da atmosfera ao redor dos frutos através da aplicação de barreiras artificiais, como filmes plásticos ou ceras, proporciona uma microatmosfera, com umidade relativa maior que a externa. Isso altera a taxa de transpiração e, consequentemente, reduzindo a perda de água, retardando mudanças no teor de açúcares, na cor, na textura, e ainda reduzindo as perdas de acidez, pela diminuição da atividade de enzimas relacionadas ao metabolismo respiratório, aumentando a vida útil destas (CHITARRA \& CHITARRA, 1990; FIGUEIREDO et al., 2007).

Dessa forma, o objetivo do presente trabalho foi avaliar a conservação pós-colheita de camu-camu sob refrigeração $\left(5 \pm 1^{\circ} \mathrm{C}\right.$ e $90 \pm 2 \%$ UR) e em diferentes tipos de embalagens, com relação aos aspectos físicos e químicos.

\section{MATERIAL E MÉTODOS}

Os frutos utilizados no experimento foram provenientes da 'Fazenda Eldorado' na região de Cajati, localizado no sul do Estado de São Paulo.
Após a colheita, os frutos foram selecionados visualmente quanto ao grau de maturação, aparência e sanidade. Utilizaram-se somente os frutos sadios e meio-maduros, ou seja, de coloração vermelho-claro púrpura. Após a seleção, os frutos foram pesados e acondicionados em bandejas de polipropileno (PP) (Delpak Embalagens). Cada bandeja (unidade experimental) continha em média $200 \mathrm{~g}$ de frutos, os quais receberam os seguintes tratamentos: controle (bandejas sem revestimento), PVC (bandejas revestidas com filme policloreto de vinila, $15 \mu \mathrm{m}$ de espessura, permeabilidade ao $\mathrm{O}_{2}$ e ao $\mathrm{CO}_{2}$ de 3162 e $31620 \mathrm{cc} \mathrm{m}^{-2} 24 \mathrm{~h}-1$, respectivamente) e $\mathrm{BOPP}_{50}$ (polipropileno biaxialmente orientado, $50 \mu \mathrm{m}$ de espessura, permeabilidade ao $\mathrm{O}_{2}$ e ao $\mathrm{CO}_{2}$ de 785 e $2355 \mathrm{cc} \mathrm{m}^{-2} 24 \mathrm{~h}^{-1}$, respectivamente), foram utilizadas quatro bandejas de cada filme em cada período de análise. Em seguida, as amostras foram colocadas em câmara frigorífica (Prófrio Refrigeração Industrial) com temperatura de $5 \pm 1^{\circ} \mathrm{C}$ e $90 \pm 2 \%$ de umidade relativa (UR), na qual permaneceram armazenadas por 25 dias. A avaliação dos frutos ocorreu um dia após a aplicação dos tratamentos e posteriormente a cada 4 dias de armazenamento, ou seja, nos dias 1, 5, $9,13,17,21$ e 25 .

O teor de sólidos solúveis foi determinado em refratômetro Auto Abbe, modelo 10500/10501, Leica, segundo método 932.12 da AOAC (2005). $\mathrm{O} \mathrm{pH}$ foi determinado em potenciômetro da marca MARCONI, segundo método $n^{0} 981.12$ da AOAC (2005) e acidez titulável, expressa em percentual de ácido cítrico, segundo o método ํo $942.15 \mathrm{~B}$ da AOAC (2005).

O teor de ácido ascórbico foi determinado pelo método de STROHECKER \& HENNING (1967), que consiste na redução de 2,6-diclorofenolindofenolsódio (DFNa) pelo ácido ascórbico. Em meio alcalino, o DFNa é azul, em meio ácido, é rosa e sua forma reduzida é incolor. Dessa forma, o ponto final da titulação foi detectado pela viragem da solução de incolor para rosa. Os resultados foram expressos em $g$ de ácido ascórbico $100 \mathrm{~g}^{-1}$ de camu-camu.

A quantificação das antocianinas totais foi realizada segundo método $\mathrm{n}^{\mathrm{o}} 2005.02$ (técnica de diferença de $\mathrm{pH}$ ) da AOAC (2005). A polpa homogeneizada de camu-camu foi centrifugada a $8.000 \mathrm{rpm} \mathrm{a} 4^{\circ} \mathrm{C}$ por 20 minutos. Após a centrifugação, retirou-se o sobrenadante e colocou-se em tubos de rosca. As amostras foram preparadas em tubos de ensaio, havendo dois preparos de solução, uma com adição de $1,0 \mathrm{~mL}$ de solução tampão cloreto de potássio $\mathrm{pH} 1,0(\mathrm{KCl} 0,025 \mathrm{M})$ e $1,5 \mathrm{~mL}$ da polpa centrifugada; e a outra com 1,0 mL de solução tampão 
acetato de sódio $\mathrm{pH} 4,5\left(\mathrm{CH}_{3} \mathrm{CO}_{2} \mathrm{Na} \cdot 3 \mathrm{H}_{2} \mathrm{O}\right.$ 0,4M) em $1,5 \mathrm{~mL}$ da polpa centrifugada. Realizaram-se leituras em espectrofotômetro com absorbância a $520 \mathrm{~nm}$ e a absorbância $700 \mathrm{~nm}$ para ambas as soluções tampão. Os resultados foram expressos em $\mathrm{mg}$, equivalente cyanidin-3-glucosideo $\mathrm{g}^{-1}$ polpa.

A Firmeza foi determinada com o auxílio do Penetrômetro Fruit Pressure Test, modelo FDN1 $(0,1$ a $1 \mathrm{~N})$, com ponteira de $6 \mathrm{~mm}$, tomando-se uma leitura da região equatorial de cada fruto. As medidas foram convertidas em unidades de Newton, multiplicando cada medida por 9,82 N (COELHO, 1994). A Perda de Massa foi determinada através da diferença entre a massa inicial e a massa final da amostra em balança semi-analítica (Micronal, modelo B3600), com precisão de $0,01 \mathrm{~g}$, sendo os resultados expressos em porcentagem (\%). Para se determinar a concentração de $\mathrm{CO}_{2}$, coletaram-se amostras de 1 $\mathrm{mL}$ de gás do interior das embalagens, através de um septo de silicone presente na superfície delas e com o auxílio de uma seringa acoplada a um analisador de gases portátil PBI Dansensor, os resultados foram expressos em porcentagem de $\mathrm{CO}_{2}$

$\mathrm{O}$ delineamento experimental foi $\mathrm{O}$ inteiramente aleatorizado em esquema fatorial duplo, sendo as fontes de variação, três tipos de embalagens (controle, PVC e $\mathrm{BOPP}_{50}$ ) versus sete tempos de análises $(1,5,9,13,17,21$ e 25 dias de armazenamento) com quatro repetições. Os resultados obtidos a partir das avaliações físicas e químicas foram submetidos à análise de variância (ANOVA) pelo Teste F, comparação das médias pelo Teste de Tukey (5\%), utilizando-se o sistema estatístico Statistical Analysis System (SAS, 1996). Para as análises de perda de massa e concentração de $\mathrm{CO}_{2}$, realizou-se o ajustamento a modelos de regressão, os quais seguiram o coeficiente de determinação $\left(\mathrm{R}^{2}\right)$.

\section{RESULTADOS E DISCUSSÃO}

Os frutos de camu-camu apresentaram um comportamento linear de perda de massa ao longo dos dias de armazenamento, sendo que os revestidos por filmes plásticos tiveram as menores perdas, quando comparados com aqueles sem embalagem, ou seja, 3,56 e 0,26\%, quando embalados com PVC e BOPP $_{50}$, respectivamente, e $14,24 \%$ sem embalagem (Figura 1a). A menor perda de massa dos frutos revestidos se deve ao fato da atmosfera modificada permitir a formação de um microambiente saturado de umidade, possibilitando a redução do gradiente de pressão de vapor entre o produto e a atmosfera interna da embalagem, diminuindo a transpiração dos frutos (FONSECA et al., 2000; SOUSA et al., 2000). Após 15 dias de armazenamento, por meio de avaliação visual, os frutos sem revestimentos já não apresentavam uma aparência recomendável para a comercialização, devido à intensa desidratação, que ocasionou o enrugamento da casca. A evaporação e a não reposição da água perdida nos tecidos dos produtos hortícolas resulta em importantes perdas de peso (valor comercial), alterações na aparência dos frutos, como murchamento e enrugamento, além de perdas na qualidade textural e nutricional (AKED, 2002; SCALON et al., 2004). O segundo tratamento que apresentou a maior taxa de perda de massa (3,56\%) foi o de PVC, porém esse percentual foi insuficiente para gerar algum dano na aparência dos frutos, mantendo-os viáveis para comercialização por 21 dias, já que, após esse período, ocorreu a incidência de fungos.

MACHADO et al. (2007) e AGOSTINI et al. (2009), ao analisarem jabuticabas armazenadas em diferentes condições de embalagens e temperaturas, também observaram maior perda de massa em frutos não revestidos por filmes plásticos. Em morangos, a perda de massa também foi verificada ao longo do armazenamento por ANTUNES et al. (2003) e igualmente maiores perdas foram constatadas em frutos sem atmosfera modificada por CALEGARO et al. (2002), comprovando a eficiência da utilização de filmes plásticos na manutenção da massa fresca dos frutos.

A menor perda de massa observada nos frutos revestidos com $\mathrm{BOPP}_{50}(0,26 \%)$ em relação ao $\mathrm{PVC}$ pode ser associada à menor permeabilidade daquele filme ao vapor de água. A redução da perda de massa está diretamente relacionada à taxa de transmissão de vapor d'água da embalagem, ou seja, quanto menor a taxa de transmissão, menor o déficit de pressão de vapor d'água e maior a umidade relativa no interior da embalagem, reduzindo a taxa de transpiração das frutas (SANCHES et al., 2011).

A concentração de $\mathrm{CO}_{2}$ no interior das embalagens revestidas por filme de PVC foi constante ao longo do armazenamento, ficando em torno de $2,62 \%$ de $\mathrm{CO}_{2}$ (Figura 1 b). Já o acondicionamento do camu-camu em $\mathrm{BOPP}_{50}$ causou rápido acúmulo de 


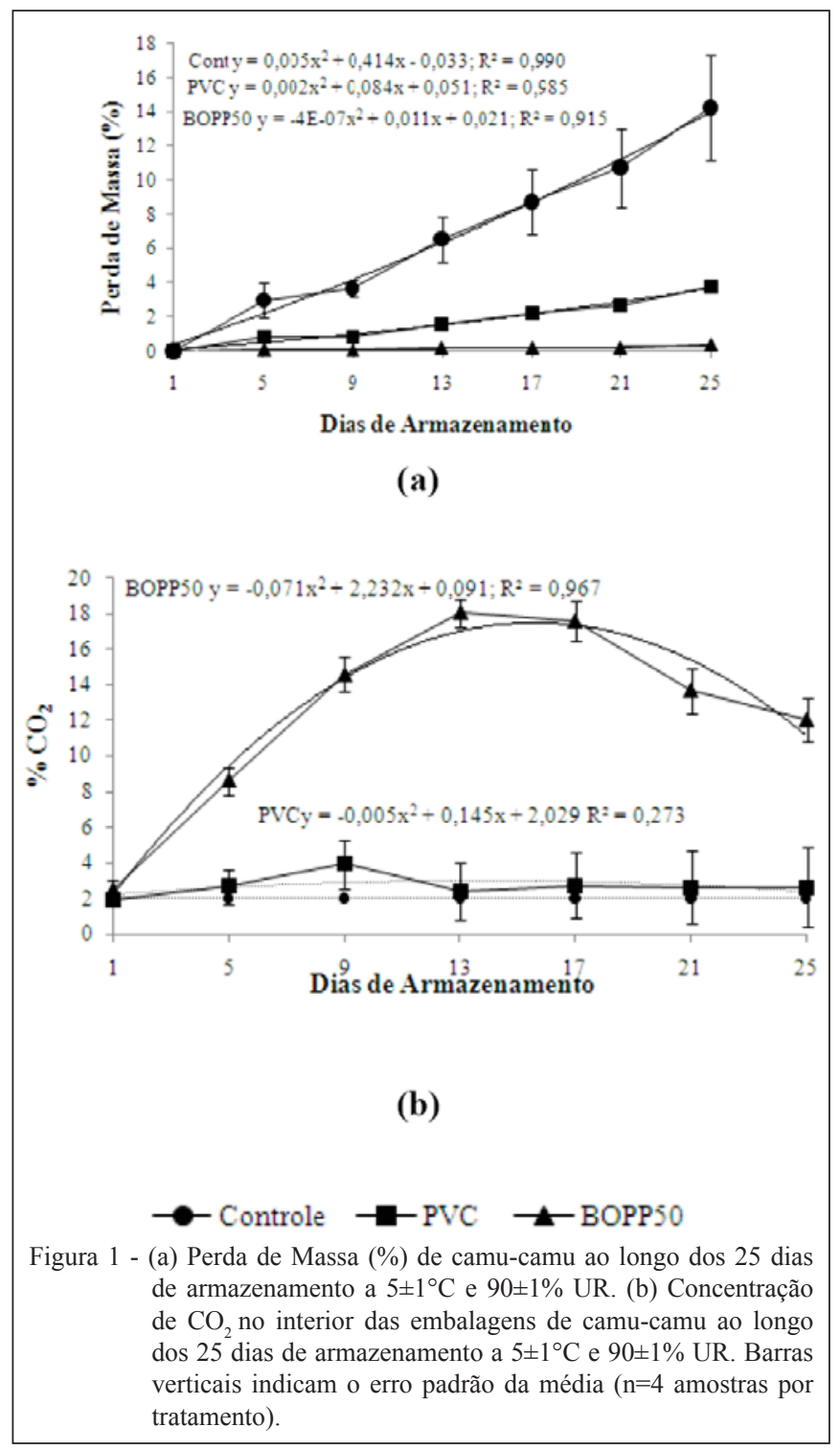

$\mathrm{CO}_{2}$ até o $13^{\circ}$ dia de armazenamento, o que pode ser justificado pela menor permeabilidade do filme a este gás. Com isso, essas amostras apresentaram, com 13 dias de armazenamento, o estufamento da embalagem, exsudação dos frutos e odor característico de etanol, indicando a ocorrência de condições anaeróbicas. Os frutos em condições de anaerobiose elevam a produção de acetaldeído que, em altas concentrações, tornam-se tóxicos para células vegetativas, assim, por meio adaptativo, ele é rapidamente transformado em uma substância menos tóxica, o etanol, através da enzima álcool dehidrogenase (HRIBAR et al., 2000). Segundo SAQUET \& STREIF (2000), tanto o acetaldeído quanto o etanol podem desencadear o surgimento de degenerescência da polpa dos frutos, além da formação de sabores e odores alcoolicos, não característicos dos frutos.

O teor de sólidos solúveis não variou significativamente entre as embalagens. Somente no $25^{\circ}$ dia de armazenamento, os frutos do controle diferiram dos demais com a maior média, conforme mostra a tabela 1. AGOSTINI et al. (2009), ao avaliarem a conservação de jabuticabas em diferentes condições de embalagens e temperaturas, notaram que o controle (frutos não embalados) apresentou os maiores teores de sólidos solúveis. Esse fato pode ser justificado pela perda da turgescência com consequente concentração da polpa, devido à maior perda de água neste tratamento. SCALON et al. (2004), em estudo de conservação pós-colheita de 
Tabela 1 - pH, acidez titulável (\% ácido cítrico) e sólidos solúveis ( ${ }^{\circ} \mathrm{Brix}$ ) em frutos de camu-camu armazenados a $5 \pm 1{ }^{\circ} \mathrm{C}$ e $90 \pm 1 \%$ UR sem embalagem e embalados com PVC e BOPP 50 (valores médios, $\pm \mathrm{DP}, \mathrm{n}=4$ ).

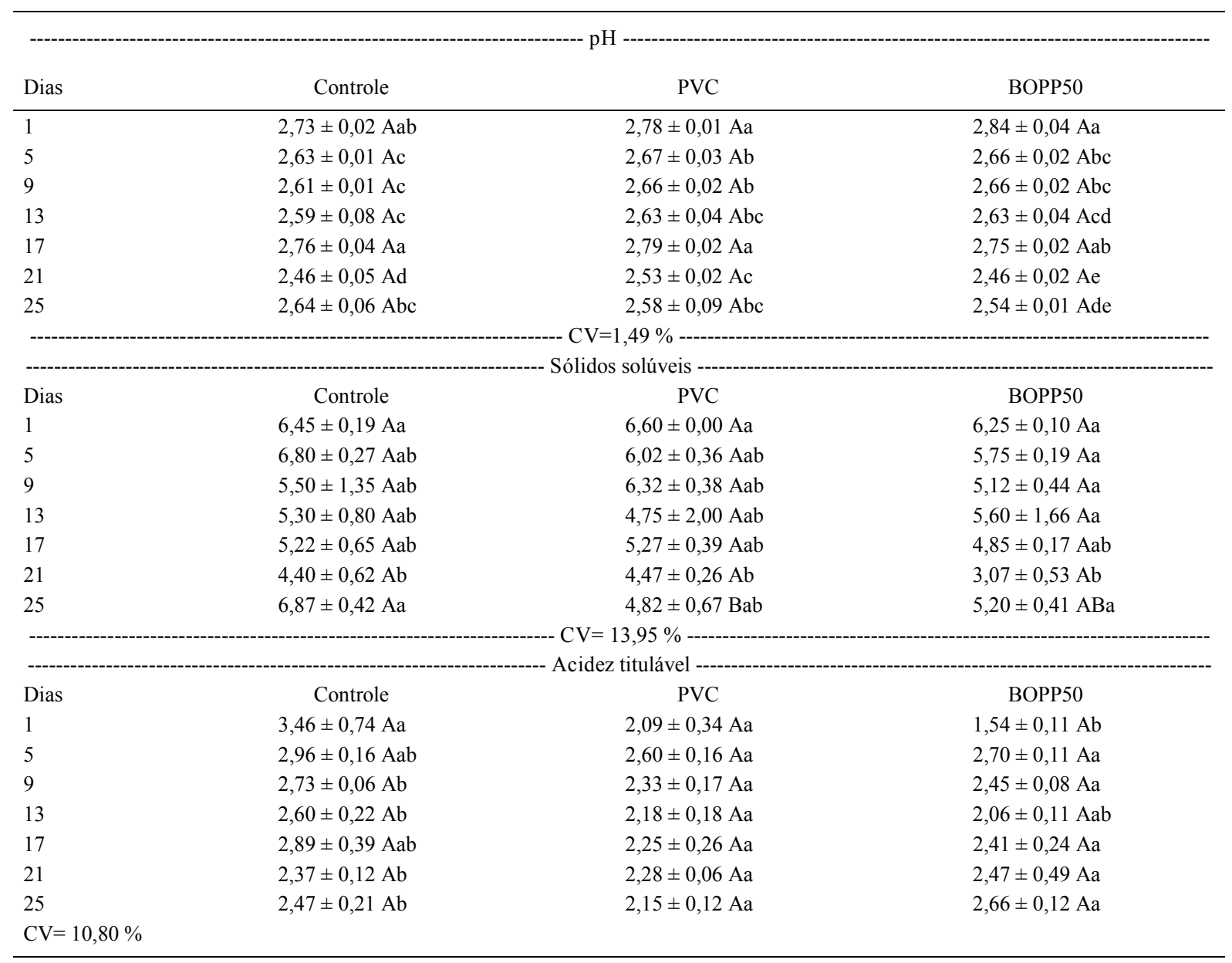

Controle - frutos sem revestimentos de filmes; $\mathrm{PVC}$ - policloreto de vinil; $\mathrm{BOPP}_{50}$ - polipropileno biaxialmente orientado; $\mathrm{DP}=\mathrm{Desvio}$ Padrão das médias; $n=$ número de amostras utilizadas em cada tratamento; $C V=$ Coeficiente de variação.

Nota: Médias seguidas pela mesma letra maiúscula na linha e minúscula na coluna não diferem entre si em nível de $5 \%$ de probabilidade, pelo Teste de Tukey.

uvaia, observaram que as diferentes embalagens avaliadas também não influenciaram de forma significativa os teores de sólidos solúveis. Mediante a observação dos resultados obtidos para o parâmetro firmeza, pode-se inferir que as diferenças constatadas até o 9o dia de armazenamento, independente da embalagem utilizada, estejam relacionadas com a variabilidade da matéria-prima, já que, em alguns dias, os valores aumentaram e, em outros, diminuíram sem apresentar comportamento coerente (Figura 2). A partir do $13^{\circ}$ dia, foi possível notar que a firmeza dos frutos se caracterizou pelo decréscimo gradual com o avanço dos dias de armazenamento, principalmente no tratamento com o filme de menor permeabilidade
$\left(\mathrm{BOPP}_{50}\right)$ em que a perda chegou a $59 \%$ no $21^{\circ}$ dia, enquanto o tratamento controle e o PVC apresentaram perdas de 30 e $25 \%$, respectivamente. A maior perda de firmeza observada no tratamento com filme de $\mathrm{BOPP}_{50}$ pode ser associada à maior concentração de $\mathrm{CO}_{2}$ presente no interior da embalagem. Segundo KADER et al. (1989), elevadas taxas desse gás causam injúrias nos tecidos vegetais, manifestandose como amadurecimento irregular, aumento da biossíntese de etileno, aceleração da deterioração e agravamento de desordens fisiológicas.

O pH e a acidez titulável (Tabela 1) não variaram significativamente entre os tipos de embalagens utilizadas, mas sim entre os dias de 


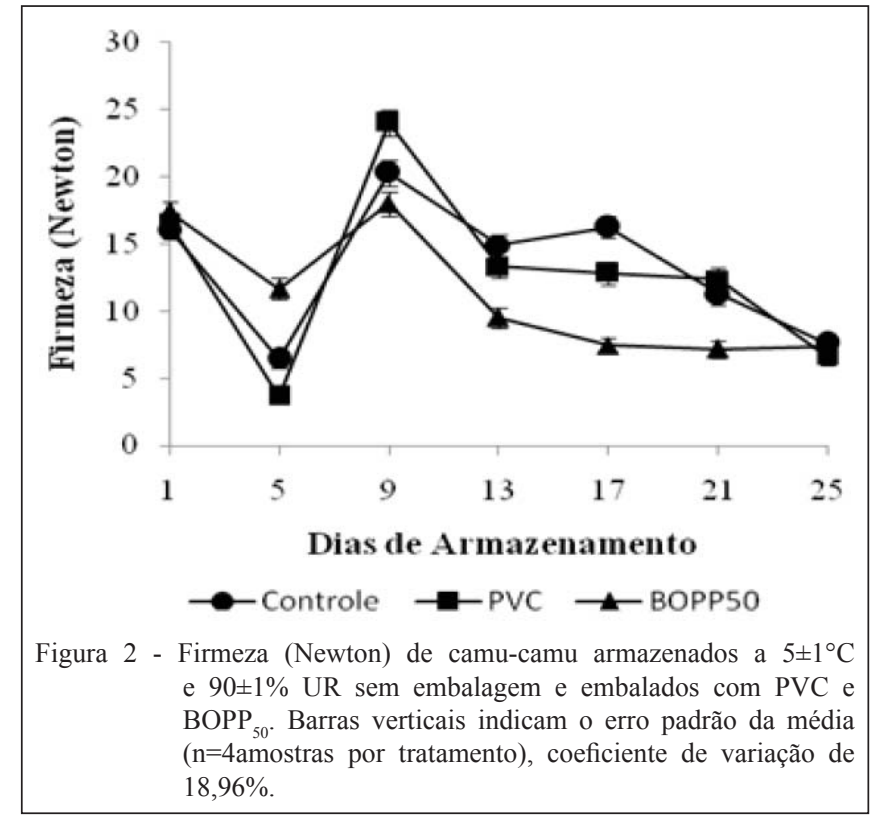

armazenamento. ANTUNES et al. (2003) e CIA et al. (2007), trabalhando com amora-preta sob atmosfera modificada e ambiente refrigerado, também não observaram alterações significativas de $\mathrm{pH}$ depois de 12 dias de armazenamento. Percebeu-se uma queda do $\mathrm{pH}$ ao longo dos dias, o que sugere a síntese de ácidos orgânicos concordante com o aumento da acidez titulável. Já as oscilações nas médias da acidez ao longo do armazenamento, segundo CHITARRA \& CHITARRA (2005), podem estar relacionadas aos processos bioquímicos do metabolismo respiratório, que tanto sintetiza quanto consome ácido como esqueleto de carbono.

O teor de ácido ascórbico não foi influenciado significativamente pelos tratamentos, tendo isso ocorrido apenas pelos dias de armazenamento em que se observou uma queda gradativa ao longo dos 25 dias (Figura 3a). CALEGARO et al. (2002) e VIEITES et al. (2006), em estudo com morangos, também verificaram diminuição nos teores de vitamina $\mathrm{C}$ durante o armazenamento. $\mathrm{O}$ menor decréscimo ocorreu no controle $(24,46 \%)$, o que pode ser associado à maior perda de água neste tratamento, que resultou na concentração desta vitamina nos frutos. Entretanto, a concentração de antocianinas sofreu influência significativa dos tratamentos e dos dias de armazenamento. Ocorreu uma tendência na redução deste composto ao longo dos 25 dias, sendo mais evidente no tratamento com $\mathrm{BOPP}_{50}$, em que ocorreram perdas de 48\% (Figura 3b). A elevada concentração de $\mathrm{CO}_{2}$ presente nesta embalagem pode ter acelerado a degradação das antocianinas. MOURA et al. (2005) em trabalho com pedúnculo de caju sob atmosfera modificada, observaram que a cor se manteve mais constante nas embalagens com menor concentração desse gás, indicando assim menor degradação das antocianinas.

\section{CONCLUSÃO}

Embora não tenha ocorrido variação significativa entre as embalagens para os atributos químicos do camu-camu, a aparência destes pode inviabilizar sua comercialização in natura. $\mathrm{O}$ investimento em embalagens adequadas pode evitar tanto o processo de murcha quanto o processo fermentativo dos frutos. Dessa forma, os frutos revestidos com filmes de PVC e mantidos a $5 \pm 1^{\circ} \mathrm{C}$ conservaram a aparência e a qualidade para comercialização até o $21^{\circ}$ dia de armazenamento, sendo recomendado esse tipo de embalagem para a conservação pós-colheita do camu-camu.

\section{AGRADECIMENTO}

Os autores agradecem à Fundação de Amparo à Pesquisa do Estado de São Paulo (FAPESP), pelo apoio e pela concessão da bolsa de Mestrado concedida ao primeiro autor ( $\mathrm{n}^{-}$ processo: 2010/14435-0). 


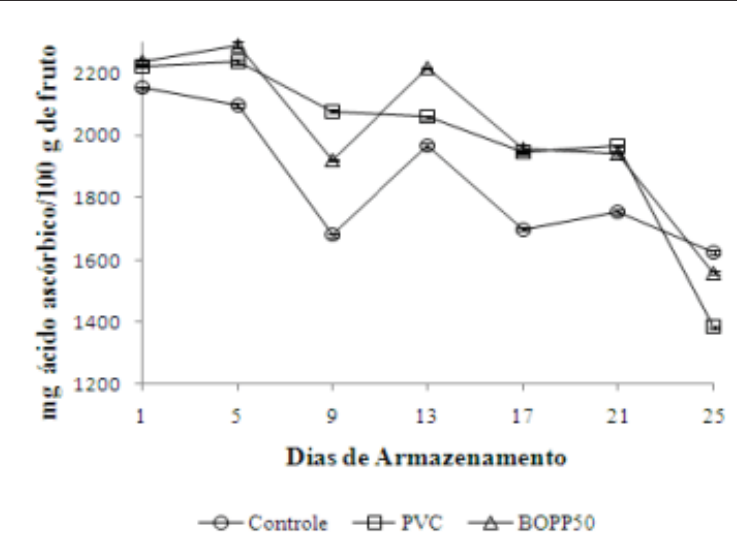

(a)

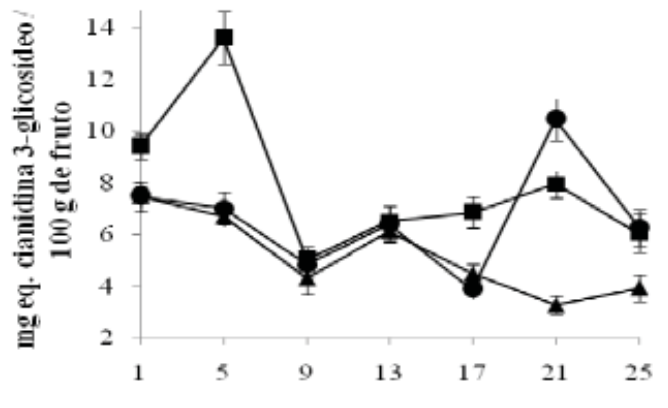

Dias de Armazenamento

(b)

\section{- Controle - -PVC - -BOPP50}

Figura 3 - (a) Vitamina C (mg ácido ascórbico/100 g de fruto) e (b) Teor de Antocianina (mg eq. Cianidina 3-glicosídeo/ $100 \mathrm{~g}$ de fruto) de camu-camu armazenados a $5 \pm 1^{\circ} \mathrm{C}$ e $90 \pm 1 \%$ UR sem embalagem e embalados com PVC e BOPP $_{50}$. Barras verticais indicam o erro padrão da média ( $\mathrm{n}=4$ amostras por tratamento); $\mathrm{CV}$ (Coeficiente de variação) vitamina $\mathrm{C}=9,25 \%$ e CV (Coeficiente de variação) antocianinas $=24,14 \%$.

\section{REFERÊNCIAS}

AGOSTINI, J.S. et al. Atmosfera modificada e condições de armazenamento nas características físico-químicas de jabuticabas da cultivar 'paulista'. Ciência Rural, Santa Maria, v.39, n.9, p.2601-2608, 2009. Disponível em: <http://www.scielo.br/pdf/cr/ v39n9/a365cr1679.pdf>. Acesso em: 4 nov. 2013. doi: 10.1590/ S0103-84782009005000205.

AKED, J. Maintaining the postharvest quality of fresh fruits and vegetables._In: JONGEN, W. (Ed.). Fruit and vegetable processing: improving quality. Boca Raton: CRC, 2000. p. 119-150.
AOAC (ASSOCIATION OF OFFICIAL ANALITICAL CHEMISTRY). Official methods of analysis of the Association of Analytical Chemists International. 18.ed. Washington, 2005. 1583p.

ANTUNES, L.E.C. et al. Conservação pós-colheita de frutos de amora-preta. Pesquisa Agropecuária Brasileira, Brasília, v.38, n.3, p.413-419, 2003. Disponível em: <http://www.scielo.br/pdf/ pab/v38n3/v38n3a11.pdf>. Acesso em: 4 nov. 2013. doi: 10.1590/ S0100-204X2003000300011.

ARÉVALO PINEDO, R. Estudo da estabilização da polpa de Camu-camu (Myrciaria dúbia (H.B.K.) Mc Vaugh) congelada

Ciência Rural, v.44, n.6, jun, 2014. 
visando à manutenção de ácido ascórbico e de antocianinas. 2007. 155f. Dissertação (Doutorado em Engenharia Química) - Faculdade de Engenharia Química, Universidade Estadual de Campinas, SP.

CALEGARO, J.M. et al. Utilização de atmosfera modificada na conservação de morangos em pós-colheita. PesquisaAgropecuária Brasileira, Brasília, v.37, n.8, p.1049-1055, 2002. Disponível em: $<$ http://www.scielo.br/pdf/pab/v37n8/11663.pdf>. Acesso em: 4 nov. 2013. doi: 10.1590/S0100-204X2002000800001.

CHITARRA, M.I.F.; CHITARRA, A.B. Pós-colheita de frutas e hortaliças: fisiologia e manuseio. Lavras: ESAL/FAEPE, 1990. 293p.

CHITARRA, M.I.F.; CHITARRA, A.B. Pós-colheita de frutas e hortaliças: fisiologia e manuseio. Lavras: UFLA, 2005. 785p.

CIA, P.; BRON, U.I. et al. Atmosfera modificada e refrigeração para conservação pós-colheita da amora-preta. Bioscience Journal, Uberlândia, v.23, n.3, p.11-16, 2007. Disponível em: <http://www. seer.ufu.br/index.php/biosciencejournal/article/view/6668/4393>. Acesso em: 4 nov. 2013.

COELHO, A.H.R. Qualidade pós-colheita de pêssegos. Informe Agropecuário, Belo Horizonte, v.17, n.180, p.31-39, 1994.

FIGUEIREDO, R.W. et al. Qualidade de pedúnculos de caju submetidos à aplicação pós-colheita de cálcio e armazenados sob refrigeração. Pesquisa Agropecuária Brasileira, Brasília, v.42, n.4, p.475-482, 2007. Disponível em: <http://seer.sct.embrapa.br/ index.php/pab/article/view/7591/4510>. Acesso em: 4 nov. 2013 doi: 10.1590/S0100-204X2007000400004.

FONSECA,S.C. et al. Modelling $\mathrm{O}_{2}$ and $\mathrm{CO}_{2}$ exchange for development of perforation-mediated modified atmosphere packaging. Journal of Food Engineering, Londres, v.43, n.1, p. 9-15, 2000. Disponível em: <http://www.sciencedirect.com/ science/article/pii/S0260877499001223 >. Acesso em: 4 nov. 2013 doi: 10.1016/S0260-8774(99)00122-3.

HRIBAR, J. et al. Changes during storing and astringency removal of persimmon fruit (Diospyros kaki L.). Acta Alimentaria, Budapeste, v.29, n.2, p.123-136, 2000. Disponível em: <https:// akkrt.metapress.com/content/w6m80m5725374304/resourcesecured/?target=fulltext.pdf\&sid=brzekcbbm0bakhjrs2pxcj5h\&s $\mathrm{h}=$ www.akademiai.com $>$. Acesso em: 4 nov. 2013. doi: 10.1556/ AAlim.29.2000.2.3.

KADER, A.A. et al. Modified atmosphere packaging of fruits and vegetables. Critical Reviews in Food Science and Nutrition, West Palm Beach, v.28, n.1, p.1-30, 1989. Disponível em: <http:// ucce.ucdavis.edu/files/datastore/234-525.pdf>. Acesso em: 4 nov. 2013. doi: 10.1080/10408398909527490.

MACHADO, N.P. et al. Embalagens plásticas e refrigeração na conservação pós-colheita de jabuticabas. Revista Brasileira de Fruticultura, Jaboticabal, v.29, n.1, p.166-168, 2007.
Disponível em: <http://www.scielo.br/scielo.php?pid=S010029452007000100034\&script $=$ sci arttext $>$. Acesso em: 4 nov. 2013. doi: 10.1590/S0100-29452007000100034.

MOURA, C.F.H. et al. Aparência e cor da película de pedúnculos de clones de cajueiro anão precoce para consumo in natura armazenados sob diferentes camadas de PVC. Revista Ciência Agronômica, Fortaleza, v.36, n.3, p.371-375, 2005. Disponível em: <http://www. ccarevista.ufc.br/seer/index.php/ccarevista/article/view/277/272>. Acesso em: 4 nov. 2013.

MYODA, T. et al. Antioxidative and antimicrobial potential of residues of camu-camu juice production. Journal of Food, Agriculture \& Environment, Helsinki, v.8, n.2, p.304-307, 2010. Disponível em: <http://world-food.net/download/journals/2010issue_2/f60.pdf $>$. Acesso em: 16 jan. 2014.

RUFINO, M.D.M. et al. Bioactive compounds and antioxidant capacities of 18 non-traditional tropical fruits from Brazil. Food Chemistry, Barking, v.121, n.4, p.996-1002, 2010. Disponível em: <http://www.sciencedirect.com/science/article/pii/ S0308814610001172>. Acesso em: 4 nov. 2013. doi: 10.1016/j. foodchem.2010.01.037

SANCHES, J. et al. Atmosfera modificada e refrigeração para conservação pós-colheita da nêspera 'Fukuhara'. Bragantia, Campinas, v. 70, n. 2, p. 455-459, 2011. Disponível em: <www. scielo.br/pdf/brag/v70n2/29.pdf>. Acesso em 4 nov. 2013.

SAQUET, A.A.; STREIF, J. Untersuchungen zur Atmung und zur Ethylenbildung einiger neuer Apfelsorten. Erwerbsobstbau, Berlim, v.42, p.109-112, 2000

SCALON, S.P.Q. et al. Temperatura e embalagens na conservação pós-colheita de Eugênia uvalha Cambess-Mirtaceae. Ciência Rural, Santa Maria, v.34, n.6, p.1965-1968, 2004. Disponível em: <www. scielo.br/pdf/cr/v34n6/a48v34n6.pdf>. Acesso em: 4 nov. 2013.

SOUSA, R.F. et al. Armazenamento de ciriguela (Spondia purpurea L.) sob atmosfera modificada e refrigeração. Revista Brasileira de Fruticultura, Jaboticabal, v.22, n.3, p.334-338, 2000

STROHECKER, R.; HENNING, H.M. Analisis de vitaminas: métodos comprobados. Madrid: Paz Montalvo, 1967. 428p.

VIEITES, R.L. et al. Conservação de morango armazenado em atmosfera modificada. Semina: Ciências Agrárias, Londrina, v.27, n.2, p.243-252, 2006. Disponível em: <http://www.uel.br/revistas/ uel/index.php/semagrarias/article/view/2426/2073>. Acesso em: 4 nov. 2013.

YUYAMA, K. A cultura de camu-camu no Brasil. Revista Brasileira de Fruticultura, Jaboticabal, v.33, n.2, p.335-690, 2011. Disponível em: $<$ http://www.scielo.br/scielo.php?pid=S0100$29452011000200001 \&$ script $=$ sci arttext $>$. Acesso em: 4 nov. 2013. doi: $10.1590 / \mathrm{S} 0100-29452011000200001$. 